DOI 10. 18307/2018. 0322

(C) 2018 by Journal of Lake Sciences

\title{
青藏高原典型湖库光学吸收特性与光合有效辐射衰减系数初步 研究
}

尚盈辛 ${ }^{1,3}$, 宋开山 ${ }^{1 * *}$, 蒋 盼 $^{1}$, 马建行 ${ }^{2,3}$, 温志丹 ${ }^{1}$,赵 莹 $^{1,3}$

(1: 中国科学院东北地理与农业生态研究所, 长春 130102)

(2: 中国科学院遥感与数字地球研究所, 北京 100094)

(3: 中国科学院大学,北京 100049)

摘 要: 青藏高原地区分布的湖泊数量众多、面积较大、分布范围广泛. 受制于恶劣的自然条件, 对该地区湖泊的光学吸 收特性以及光合有效辐射衰减系数 $\left(K_{\mathrm{d}}(\mathrm{PAR})\right)$ 的研究鲜有成果. 本文依据 2014 和 2015 年间采集的 13 个典型高海拔、 湖泊面积较大的湖库的现场实测数据和实验室测定数据, 分析了采样湖库各个采样点的 $K_{\mathrm{d}}(\mathrm{PAR})$ 特征以及有色可溶性 有机物 $(\mathrm{CDOM})$ 、藻类颗粒物吸收及非色素颗粒物吸收特性,计算并分析了 $K_{\mathrm{d}}(\mathrm{PAR})$ 与透明度以及光学活性物质的关系. 研究结果表明: 青藏高原地区各湖库平均各项颗粒物吸收系数均较低, 总颗粒物吸收系数在 $400 \sim 700 \mathrm{~nm}$ 波段内不超过 $0.14 \mathrm{~m}^{-1}$ 、CDOM 吸收系数在 $355 \mathrm{~nm}$ 波长处最高, 为 $1.23 \mathrm{~m}^{-1}$ 、最低接近于 0 、藻类颗粒物吸收特性不明显; 实验数据完整 的巴木错、格仁错和班公错的主导吸收组分各异, 其中巴木错为 CDOM 吸收主导, 格仁错与班公错为非色素颗粒物吸收 主导; 青藏高原采样湖泊总体 $K_{\mathrm{d}}(\mathrm{PAR})$ 平均值较小, 仅为 $0.26 \mathrm{~m}^{-1}$, 样点最大值出现在可鲁克湖 $\left(1.17 \mathrm{~m}^{-1}\right)$, 最小值出现 在普莫雍错 $\left(0.10 \mathrm{~m}^{-1}\right)$; 在采样湖泊中 $K_{\mathrm{d}}(\mathrm{PAR})$ 与透明度呈显著相关; $K_{\mathrm{d}}(\mathrm{PAR})$ 与 $\mathrm{CDOM}$ 的相关性最强, 叶绿素 a 浓度 次之,与总悬浮颗粒物浓度的相关性最不显著.

关键词: 青藏高原; 光学活性物质; 光学吸收特性; 光合有效辐射衰减系数

\section{Optical absorption properties and diffuse attenuation of photosynthetic active radiation for inland waters across the Tibetan Plateau}

\author{
SHANG Yingxin ${ }^{1,3}$, SONG Kaishan ${ }^{1 * *}$, JIANG Pan ${ }^{1}$, MA Jianhang ${ }^{2,3}$, WEN Zhidan ${ }^{1}$ \& ZHAO Ying ${ }^{1,3}$ \\ (1: Northeast Institute of Geography and Agroecology, Chinese Academy of Sciences, Changchun 130102, P.R.China) \\ (2: Institute of Remote Sensing and Digital Earth, Chinese Academy of Sciences, Beijing 100094, P.R.China) \\ (3: University of Chinese Academy of Sciences, Beijing 100049, P.R.China)
}

Abstract: Numerous large lakes and reservoirs are distributed in the Tibetan Plateau. Because of the adverse environment, studies for optical absorption property in lakes and especially the diffuse attenuation of photosynthetic active radiation $\left(K_{\mathrm{d}}(\mathrm{PAR})\right)$ are quite limited. Here we present multiple absorption characteristics of optically active constituents and $K_{\mathrm{d}}(\mathrm{PAR})$ collected from 13 alpine lakes in 2014 and 2015, and analyze the relationship between $K_{d}($ PAR ) and various optically active constituents. The results revealed that the absorption coefficient of total suspended matter (TSM) was less than $0.14 \mathrm{~m}^{-1}$ which was low within 400$700 \mathrm{~nm}$. The absorption coefficients of chromophoric dissolved organic matter (CDOM) in $355 \mathrm{~nm}$ reached a peak of $1.23 \mathrm{~m}^{-1}$ and achieved 0 approximately in the bottom. Algal absorption was not obvious in Tibetan lakes and reservoirs, and the dominant absorption constituent varied among Bam Co, Gering Co and Pangong Co. The absorption of CDOM was dominated for the total absorption in Bam Co, while the total absorption of Gering Co and Pangong Co was dominated by the absorption of non-algal particles. Mean value of $K_{\mathrm{d}}(\mathrm{PAR})$ was $0.26 \mathrm{~m}^{-1}$ which was low in all sites compared to the existed research results of $K_{\mathrm{d}}(\mathrm{PAR})$ in several lakes

* 国家自然科学基金项目 (41471290)、中国科学院百人计划项目 ( Y3H1061001) 和吉林省科技发展计划项目 (20150519006JH) 联合资助. 2016-11-08 收稿;2017-04-12 收修改稿. 尚盈辛 (1989 ), 女, 硕士; E-mail: goodlucksyx27@163.com.

** 通信作者; E-mail: songks@iga.ac.cn. 
and reservoirs, and the maximum and minimum of $K_{d}(\mathrm{PAR})$ were observed in Lake Keluke $\left(1.17 \mathrm{~m}^{-1}\right)$ and Lake Puma Yumco $\left(0.10 \mathrm{~m}^{-1}\right)$, respectively. Significant correlation existed between $K_{d}(\mathrm{PAR})$ and secchi disk depth, and it was also found between $K_{\mathrm{d}}(\mathrm{PAR})$ and CDOM dramatically. Correlation between $K_{\mathrm{d}}(\mathrm{PAR})$ and chlorophyll-a was not so conspicuous, and $K_{\mathrm{d}}(\mathrm{PAR})$ was not in good agreement with TSM.

Keywords: Tibetan Plateau; optically active constituents; optical absorption properties; diffuse attenuation of photosynthetic active radiation

进人水体的光会被吸收和散射,这些特性只与水体成分有关,所以被称为水体固有光学特性 ${ }^{[1]}$. 影响水 体固有光学特性的物质主要包括纯水、浮游植物、非色素悬浮物及有色可溶性有机物 ( CDOM $)^{[2]}$. 光合有效 辐射 (Photosynthetically Active Radiation, PAR) 指 400 700 nm 波长内能被绿色植物利用进行光合作用的太 阳辐射部分. 对于湖库水体, 太阳辐射在进人水体后, 会受到水中悬浮颗粒物、可溶性有机物以及浮游生物 的影响而衰减 ${ }^{[3]}$, 因此为了表征 PAR 在水体中的衰减程度,一般通过测定不同深度水层的 PAR 值来计算光 合有效辐射衰减系数 $\left(K_{\mathrm{d}}(\mathrm{PAR})\right)$, 对光线在水体中的垂直分布予以指示 ${ }^{[4]}$. 水下光场分布决定了水体中浮 游生物及沉水植物在湖泊中的分布情况, 因此, $K_{\mathrm{d}}(\mathrm{PAR})$ 对湖泊生态环境的评价具有重要意义, 在湖泊生态 修复和水质改善方面具有指导作用 ${ }^{[5]}$. 近年来国内外学者在海洋和内陆水体进行了很多固有光学特性和光 合有效辐射衰减系数的研究 ${ }^{[6]}$,但是国内对于高海拔地区的湖泊光学特性的系统研究尚有空缺.

青藏高原是地球海拔最高、面积最大、形成最晚的高原, 也是具有全球意义且非常脆弱的生态系统, 从 1960s 以来, 青藏高原地区的气温一直是逐渐升高的, 相比气温, 降水则复杂且空间变异大 ${ }^{[7]}$. 加上青藏高原特 殊的地形特征, 青藏高原对周边地区乃至全球环境的影响受到了举世关注, 所以对青藏高原的研究是科学研 究和社会经济的双重需要 ${ }^{[8]}$. 随着全球水体研究的不断发展, 作为内陆环境变化、生态系统等研究的重要载 体, 对湖泊的研究得到越来越多研究者的重视. 青藏高原地区分布有地球上海拔最高、数量最多、面积最大的 高原湖泊 ${ }^{[9]}$, 湖泊面积约占全国湖泊面积的 $49.5 \%$, 且湖泊成因复杂、类型多样, 受人类活动影响较少, 对各项 环境变化响应敏感 ${ }^{[10]}$. 湖泊水库的固有光学特性与表观光学特性是湖泊研究中重要的组成部分, 由于青藏高 原恶劣的自然因素限制、湖泊面积大、分布广、地处偏远, 使得获取该地区湖泊水库的观测资料及实时数据变 得异常艰难 ${ }^{[1-1-12]}$, 所以对青藏高原湖库的研究和认识不足, 还需要开展大量和长时间的研究.

本文根据 2014 和 2015 年青藏高原地区 13 个典型的高海拔、大面积湖库水体实测数据和实验结果, 对 青藏高原湖库水体的光学活性物质特征进行了详尽的描述, 此外, 由于不同水体影响 $K_{\mathrm{d}}(\mathrm{PAR})$ 的主导因素 不同: 不同学者研究发现, $K_{\mathrm{d}}(\mathrm{PAR})$ 与光学活性物质的关系在不同水体表现各异 ${ }^{[13]}$, 有学者利用 $K_{\mathrm{d}}(\mathrm{PAR})$ 与研究区相关性较强的光学物质参数建立模型, 一定程度上实现了对 $K_{\mathrm{d}}(\mathrm{PAR})$ 的反演 ${ }^{[14-17]}$. 本文研究分析 了青藏高原典型湖泊中 $K_{\mathrm{d}}(\mathrm{PAR})$ 与光学活性物质之间的关系, 为研究青藏高原湖库 $K_{\mathrm{d}}(\mathrm{PAR})$ 主导影响因 素提供了重要依据, 同时本研究采样湖库分布范围广, 也为青藏高原湖库湖库研究提供了有力的数据积累.

\section{1 数据与方法}

\section{1 研究区概况}

分别选取了分布在西藏自治区和青海省的 13 个典型的湖库, 湖库水体面积均在 $100 \mathrm{~km}^{2}$ 以上, 其中色 林错的湖泊面积达到了 $2391 \mathrm{~km}^{2}$. 分布高程从 $2500 \mathrm{~m}$ 至 $5000 \mathrm{~m}$ 不等, 主要分布在西藏阿里, 那曲、山南地区 及青海海南和海西地区. 湖泊盐度大类涵盖了咸水湖、淡水湖, 其中主要包括拉昂错、当惹雍错等 7 个咸水 湖, 普莫雍错、可鲁克湖等 5 个淡水湖以及龙羊峡水库.

\section{2 野外数据获取}

本文数据采集时间为 2014 年 9 月和 2015 年 6-7 月,对光合有效辐射数据采集工作共覆盖青藏高原地区 13 个湖泊 (图 1), 采样点总数 48 个, 测定指标主要包括: 光合有效辐射、水质参数、透明度、采样点 GPS 点位. 1.2.1 水样采集及现场测定 每个采样点通常采集水面以下 $0.1 \mathrm{~m}$ 的水体约 $2 \mathrm{~L}$, 水样带回实验室, 放置于 $4^{\circ} \mathrm{C}$ 的冰箱中败藏, $2 ~ 3 \mathrm{~d}$ 内完成测定; 现场用 YSI 水质仪自动获取水温、电导率、盐度、 $\mathrm{pH}$ 、浊度、总溶解固 体 (TDS) 等水质参数. 透明度 $(\mathrm{SD})$ 采用塞氏盘测定. 


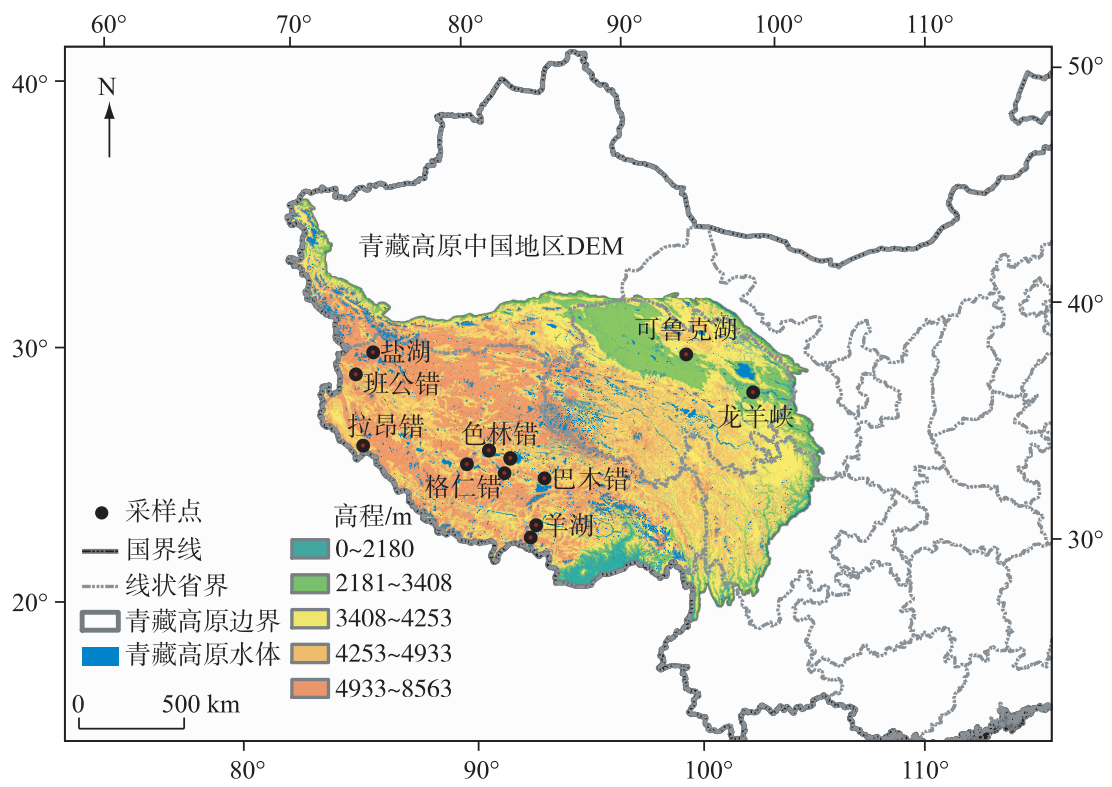

图 1 研究区地理位置及采样湖库分布

Fig. 1 Location of study area and distribution of sampling sites

1.2.2 $K_{\mathrm{d}}(\mathrm{PAR})$ 选用 Li-Cor 193SA 水下光量子仪测定水下不少于 5 个水层深度的 PAR 值.

$$
\operatorname{PAR}(Z)=\operatorname{PAR}\left(0^{-}\right) \cdot \exp \left[-K_{\mathrm{d}}(\mathrm{PAR}) \cdot Z\right]
$$

通常利用不同深度的测量值, 其中 0 代表恰好在水面以下, $Z$ 代表水深, 根据朗伯比尔定律, 利用公式 (1) 进行指数拟合, 得到 $K_{\mathrm{d}}(\mathrm{PAR})$. 要求测量深度数量 $N \geqslant 5$ 、拟合系数 $R^{2} \geqslant 0.95$ 时, 得到的拟合结果 $K_{\mathrm{d}}(\mathrm{PAR})$ 才 能被接受.

\section{3 实验室数据获取}

1.3.1 叶绿素a 浓度与总悬浮颗粒物浓度 通过 $90 \%$ 的丙酮溶液萃取, 并用 UV-2600 紫外分光光度计分别测 定 630、647、664 和 $750 \mathrm{~nm}$ 处的吸光度,进而计算出叶绿素 a(Chl.a) 浓度. 总悬浮物浓度采用称重法进行 测定.

1.3.2 CDOM 吸收系数 $\left(a_{\mathrm{CDOM}}(\lambda)\right)$ 将水样经过 $0.22 \mu \mathrm{m}$ 的聚碳酸酯表面滤膜过滤后, 利用紫外分光光度计 测定滤液在 200 $800 \mathrm{~nm}$ 的吸光度 $\mathrm{OD}(\lambda)$, 再通过公式 (2) 计算各波长处的吸收系数为消除过滤液中残留 细小颗粒物的散射, 以 $\lambda_{0}$ 为 $700 \mathrm{~nm}$ 的吸收值作为零值波段范围的参考值,利用公式(3) 做校正.

$$
\begin{gathered}
a_{\text {CDOM }}^{\prime}(\lambda)=2.303 \frac{\mathrm{OD}(\lambda)}{L} \\
a_{\mathrm{CDOM}}(\lambda)=a_{\text {CDOM }}^{\prime}(\lambda)-a_{\text {CDOM }}^{\prime}\left(\lambda_{0}\right) \cdot \frac{\lambda}{\lambda_{0}}
\end{gathered}
$$

1.3.3 总悬浮颗粒物、非色素颗粒物和藻类颗粒物吸收系数 总悬浮颗粒物吸收系数 $\left(a_{\mathrm{p}}(\lambda)\right)$ 、非色素颗粒 物吸收系数 $\left(a_{\mathrm{d}}(\lambda)\right)$ 和藻类颗粒物吸收系数 $\left(a_{\mathrm{ph}}(\lambda)\right)$ 通过定量滤膜技术获得. 利用 $0.7 \mu \mathrm{m}$ 的玻璃纤维微孔 滤膜过滤水样, 然后以空白滤膜作为参比, 利用紫外分光光度计测定其在 $300 \sim 800 \mathrm{~nm}$ 的吸光度, 最后利用 公式 (4) (6) 计算吸收系数:

$$
\begin{gathered}
\mathrm{OD}_{\mathrm{f}}(\lambda)=\mathrm{OD}(\lambda)-\frac{1}{11} \sum_{i=\lambda_{0}-5}^{\lambda_{\mathrm{o}}+5} \mathrm{OD}(i) \\
\mathrm{OD}_{\mathrm{s}}(\lambda)=0.392 \mathrm{OD}_{\mathrm{f}}(\lambda)+0.655 \mathrm{OD}_{\mathrm{f}}(\lambda)^{2} \\
a_{\mathrm{p}}(\lambda)=2.303 \frac{S}{V} \cdot \mathrm{OD}_{\mathrm{s}}(\lambda)
\end{gathered}
$$


计算时首先利用公式 (4) 对吸光度 $\mathrm{OD}(\lambda)$ 进行散射校正, 得到吸光度 $\mathrm{OD}_{\mathrm{f}}(\lambda), \lambda_{0}$ 通常取 $750 \mathrm{~nm}$; 然后 利用公式 $(5)$ 对 $\mathrm{OD}_{\mathrm{f}}(\lambda)$ 做光程放大效应的校正得到 $\mathrm{OD}_{\mathrm{s}}(\lambda)$; 最后通过公式 $(6)$ 计算得到 $a_{\mathrm{p}}(\lambda)$, 其中 $S$ 为 沉积在滤膜上颗粒物的有效面积 (单位为 $\mathrm{cm}^{2}$ ); $V$ 为过滤的水样体积 (单位为 $\mathrm{ml}$ ).

用次氯酸钠 $(\mathrm{NaClO})$ 将滤膜上的色素漂白、溶解. 以空白滤膜作为参比,测量滤膜在 $300 \sim 800 \mathrm{~nm}$ 的吸 光度, 可以得到 $a_{\mathrm{d}}(\lambda) . a_{\mathrm{ph}}(\lambda)$ 为 $a_{\mathrm{p}}(\lambda)$ 与 $a_{\mathrm{d}}(\lambda)$ 的差值.

\section{2 结果与分析}

\section{1 青藏高原典型湖库的 $K_{\mathrm{d}}(\mathrm{PAR})$ 特征}

采样的 13 个湖库 $K_{\mathrm{d}}(\mathrm{PAR})$ 计算结果 (表 1$)$ 显示, 青藏高原湖库的 $K_{\mathrm{d}}(\mathrm{PAR})$ 整体数值较低, 说明该地区 多数湖库对太阳辐射透射率高, 湖水观感上非常清澈, 水中吸光颗粒物也较少. 采样湖库中, $K_{\mathrm{d}}(\mathrm{PAR})$ 最小 的前 3 个湖库分别是普莫雍错 $\left(0.110 \mathrm{~m}^{-1}\right)$ 、当惹雍错 $\left(0.120 \mathrm{~m}^{-1}\right)$ 和羊湖 $\left(0.120 \mathrm{~m}^{-1}\right)$, 最大的 3 个采样湖库 分别是达则错 $\left(0.260 \mathrm{~m}^{-1}\right)$ 、龙羊峡水库 $\left(0.400 \mathrm{~m}^{-1}\right)$ 和可鲁克湖 $\left(0.670 \mathrm{~m}^{-1}\right)$, 其中有 10 个采样湖库的 $K_{\mathrm{d}}$ (PAR) 平均值在 $0.2 \mathrm{~m}^{-1}$ 以下; 这个结果同已有研究结果的湖库 (青藏地区之外) 在数值上相比也是较小的 (表 2), 青藏高原湖泊的 $K_{\mathrm{d}}(\mathrm{PAR})$ 与东北和华北地区的相比, 数值明显较低. 可见, 不同地区 $K_{\mathrm{d}}(\mathrm{PAR})$ 的差 异性较为明显.

表 1 采样湖库的 $K_{\mathrm{d}}(\mathrm{PAR})$ 计算结果

Tab.1 Results of $K_{\mathrm{d}}(\mathrm{PAR})$ value in sampling sites

\begin{tabular}{lccccc}
\hline \multirow{2}{*}{ 湖库名称 } & \multicolumn{2}{c}{$K_{\mathrm{d}}(\mathrm{PAR}) / \mathrm{m}^{-1}$} & \multirow{2}{*}{ 采样点数 $N$} \\
\cline { 2 - 4 } & 均值 & 标准差 & 最大值 & 最小值 & 2 \\
\hline 格仁错 & 0.200 & - & 0.230 & 0.170 & 7 \\
羊湖 & 0.120 & 0.012 & 0.140 & 0.100 & 3 \\
色林错 & 0.180 & 0.030 & 0.220 & 0.160 & 2 \\
普莫雍错 & 0.110 & - & 0.120 & 0.100 & 5 \\
可鲁克湖 & 0.670 & 0.303 & 1.170 & 0.440 & 3 \\
达则错 & 0.260 & 0.024 & 0.280 & 0.240 & 3 \\
吴如错 & 0.120 & 0.006 & 0.130 & 0.120 & 2 \\
巴年错 & 0.160 & - & 0.230 & 0.150 & 11 \\
龙羊峡水库 & 0.400 & 0.031 & 0.460 & 0.360 & 3 \\
当惹雍错 & 0.120 & 0.002 & 0.122 & 0.118 & 3 \\
拉昂错 & 0.159 & 0.003 & 0.161 & 0.156 & 3 \\
班公错 & 0.153 & 0.002 & 0.156 & 0.152 & 1 \\
错鄂湖 & 0.170 & - & - & 0.100 & 48 \\
所有样点 & 0.260 & 0.197 & 1.170 & 0 \\
\hline
\end{tabular}

表 2 部分湖库 $K_{\mathrm{d}}(\mathrm{PAR})$ 的相关研究结果

Tab.2 Existing results of $K_{\mathrm{d}}(\mathrm{PAR})$ researches in several lakes and reservoirs

\begin{tabular}{|c|c|c|c|c|}
\hline 研究区 & 水体 & 数据获取时间 & $K_{\mathrm{d}}(\mathrm{PAR}) / \mathrm{m}^{-1}$ & 来源 \\
\hline 东北地区 & 20 个典型湖库 & 2015 年 4 月－ 2015 年 9 月 & $0.47 \sim 13.93$ & 文献 $[22]$ \\
\hline 华东长三角地区 & 太湖 & $\begin{array}{l}2004 \text { 年 } 10 \text { 月 } 20 \text { 日 } \\
2004 \text { 年 } 10 \text { 月 } 29 \text { 日 }\end{array}$ & $\begin{array}{c}0.87 \sim 12.43 \\
4.42 \pm 2.64\end{array}$ & 文献 $[5]$ \\
\hline 华东闽南丘陵地区 & 晋江下游 & 2008 年 3 月 5 日 & $0.55 \sim 10.71$ & 文献 [23] \\
\hline 西北青藏高原地区 & 纳木错 & - & 0.14 & 文献 $[24]$ \\
\hline 西南云贵高原地区 & 洱海 & $\begin{array}{c}2014 \text { 年 } 4 、 7 、 11 \text { 月和 } \\
2015 \text { 年 } 1 \text { 月 }\end{array}$ & $0.42 \sim 1.45$ & 文献 $[25]$ \\
\hline
\end{tabular}


$K_{\mathrm{d}}(\mathrm{PAR})$ 平均值最高的可鲁克湖位于柴达木盆地东北缘德令哈盆地, 与其外泄相连形成的托素湖周围 是广阔的戈壁滩, 而巴音河的水常年携带上游牧民生活用水, 并裹挟着大量的牛羊粪等多种有机物注人湖 内 $^{[18]}$, 使湖底沉积物变得富含养分、泥质肥厚、杂草丛生, 尤其是浮游生物极其丰富, 而这些都是鱼类天然的 饲料. 可鲁克湖相关资料显示早在 1973 年, 当地相关部门就开始对可鲁克湖进行渔业等水产资源的开发, 受人类影响时间点较其他采样湖泊更早 ${ }^{[19]}$. 可鲁克湖较高的 $K_{\mathrm{d}}(\mathrm{PAR})$ 值与这些有关. $K_{\mathrm{d}}(\mathrm{PAR})$ 平均值最低 的普莫雍错是 13 个采样湖库中海拔最高的湖泊, 靠近喜马拉雅山主山嵴北坡, 主要依靠南面山区库拉岗日 雪山的溶雪补给, 同时还包括地下水补给, 常年人湖水量充沛 ${ }^{[20]}$, 又地处山南地区雨影地带, 降水量并不贵 乏, 属于淡水湖 ${ }^{[21]}$. 通往普莫雍错的主干道路省道 307 至 2015 年才全线贯通, 在 13 个采样湖库中属于较晚 通车的湖泊, 因而旅游及各项资源开发还未成型, 普莫雍错 $K_{\mathrm{d}}(\mathrm{PAR})$ 的较低值可能和这些有关.

\section{2 青藏高原典型湖库的光学活性物质吸收特性}

2.2.1 $a_{\mathrm{p}}(\lambda) \quad a_{\mathrm{p}}(\lambda)$ 无显著的吸收峰、谷 (图 $2 \mathrm{a}$ ). 对已测定 $a_{\mathrm{p}}(\lambda)$ 的 11 个湖库 (龙羊峡水库与可鲁克湖数据 空缺), 分别求出每个湖库 $a_{\mathrm{p}}(\lambda)$ 的平均值 $\left(a_{\mathrm{p} \_\mathrm{avg}}(\lambda)\right)$ (图 $2 \mathrm{~b}$ ). 总体来看, 在 $400 \sim 700 \mathrm{~nm}$ 波段内, 西藏地区 11 个湖库的 $a_{\text {p_avg }}(\lambda)$ 皆在很低的范围中, 最高值不超过 $0.14 \mathrm{~m}^{-1}$, 同目前已有研究的其他湖库 (吉林省湖 库 $^{[26]}$ 、辽河水体 ${ }^{[27]}$ 、东北湖库 ${ }^{[22]}$ 、太湖 ${ }^{[3,5,28]}$ 、巢湖 ${ }^{[29]}$ 、兴凯湖 ${ }^{[30]}$ 等) 相比较低. 此外, 11 个湖库中,除了格仁 错的 $a_{\mathrm{p} \_ \text {avg }}(\lambda)$ 在 $675 \mathrm{~nm}$ 特征波段出现较小的吸收峰外,其余湖库的 $a_{\mathrm{p} \_ \text {avg }}(\lambda)$ 在 440 和 $675 \mathrm{~nm}$ 及其他波段 都未观察到显著的吸收峰.
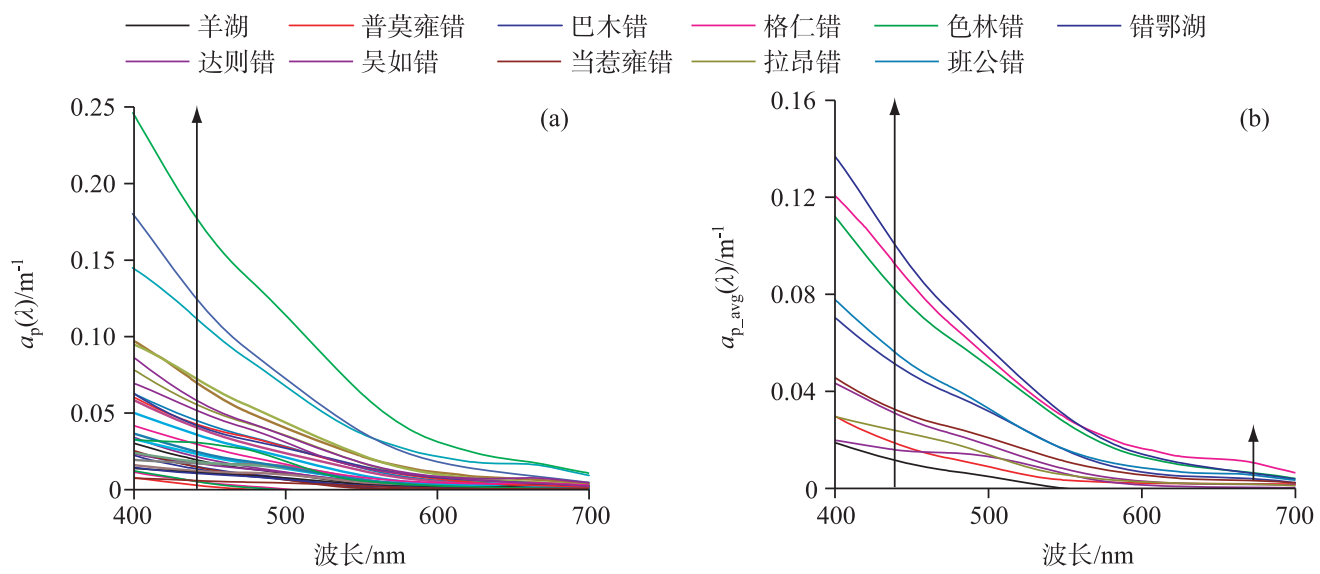

图 2 采样点的总颗粒物吸收系数

Fig.2 Absorption coefficient of total suspended matter in sampling sites

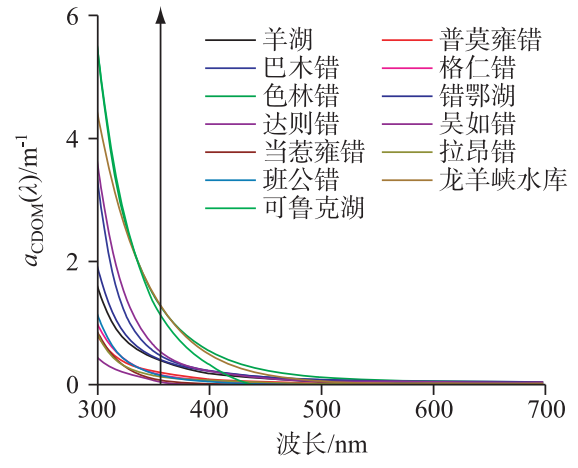

图 3 采样点的 CDOM 吸收系数

Fig.3 Absorption coefficient of CDOM in sampling sites
$2.2 .2 a_{\mathrm{CDOM}}(\lambda)$ 在不考虑人类因素影响的条件下, $\mathrm{CDOM}$ 本身属于光合作用的副产物, 在水环境中主要由腐 殖物质产生. 对于青藏高原地区大部分湖库来说, 湖库中 的 CDOM 主要由水体中的浮游生物产生. 在前人研究中, 通常用 $355 \mathrm{~nm}$ 处 CDOM 的吸收系数代表 CDOM 含量 ${ }^{[31]}$. 研究发现, 在 $355 \mathrm{~nm}$ 处所有采样湖库的 $a_{\mathrm{CDOM}}(\lambda)$ 都小于 $2 \mathrm{~m}^{-1}$ (图 3). $a_{\mathrm{CDOM}}(\lambda)$ 大于 $1 \mathrm{~m}^{-1}$ 的分别是色林错 $(1.23 \pm$ $\left.0.98 \mathrm{~m}^{-1}\right)$ 、龙羊峡水库 $\left(1.23 \pm 0.06 \mathrm{~m}^{-1}\right)$ 和可鲁克湖 $(1.09 \pm$ $0.08 \mathrm{~m}^{-1}$ ), 其余由大到小依次是达则错、巴木错、错鄂湖、 羊湖、普莫雍错、格仁错、班公错、拉昂错、当惹雍错和吴 如错. 值得注意的是, 由于青藏高原地区水体整体清澈, CDOM 含量较低, 有些甚至微乎其微, 导致个别湖库的 CDOM 过滤受到外界影响程度较大且吸收系数测定结果 
噪声较多.

2.2.3 $a_{\mathrm{d}}(\lambda)$ 和 $a_{\mathrm{ph}}(\lambda)$ 在进行吸收系数实验室测定的过程中, 由于野外采集水样总量少, 故而在 $a_{\mathrm{d}}(\lambda)$ 和 $a_{\mathrm{ph}}(\lambda)$ 测定中, 只保存了巴木错、格仁错和班公错 3 个湖库的 3 个样本数据, 所以此处为了与下文 3 个湖库 的其他吸收系数特征进行对比分析, 将这 3 个湖库对应采样点编号的 $a_{\mathrm{p}}(\lambda)$ 和 $a_{\mathrm{CDOM}}(\lambda)$ 数据提取出来 (图 $4 \mathrm{a}$ 和图 $4 \mathrm{~d}) . a_{\mathrm{p}}(\lambda)$ 在 $440 \mathrm{~nm}$ 处无明显吸收峰, 说明藻类颗粒物吸收较少, 非色素颗粒物吸收表现较为明 显, $a_{\mathrm{p}}(\lambda)$ 由高至低依次为格仁错、巴木错和班公错; 在 $675 \mathrm{~nm}$ 处 $a_{\mathrm{p}}(\lambda)$ 由高至低依次为格仁错、班公错和巴 木错, 存在轻微的吸收峰, 表现出藻类贡献的吸收特征, 其中班公错的吸收峰比格仁错更为明显, 而巴木错 不明显, 说明三者之中, 班公错中藻类颗粒物的吸收占总颗粒物吸收的比例更大, 格仁错与巴木错依次次之. 需要注意的是, 尽管 3 个湖泊在 $675 \mathrm{~nm}$ 处存在轻微的吸收峰, 但整体来看, 无论是在 $440 \mathrm{~nm}$ 处, 还是 $675 \mathrm{~nm}$ 处, 3 个湖泊峰型并不明显, 且在 $550 \mathrm{~nm}$ 处无明显的吸收谷, 说明非色素颗粒物在总颗粒物吸收中占有一定 比例. 吸收峰或吸收谷越不明显, 则说明非色素颗粒物的吸收占总吸收的比例越大.

巴木错的 $a_{\mathrm{CDOM}}(\lambda)$ 在 $300 \sim 700 \mathrm{~nm}$ 波段内远远大于格仁错与班公错. 在 $355 \mathrm{~nm}$ 处, 巴木错的 $a_{\mathrm{CDOM}}(\lambda)$ $\left(0.46 \pm 0.09 \mathrm{~m}^{-1}\right)$ 最高, 班公错 $\left(0.15 \pm 0.03 \mathrm{~m}^{-1}\right)$ 次之, 格仁错 $\left(0.13 \pm 0.03 \mathrm{~m}^{-1}\right)$ 最小, 这说明了巴木错的 CDOM 含量最高,格仁错最低 (图 4d).

可以发现在特征波长 400 和 $440 \mathrm{~nm}$ 处,格仁错的 $a_{\mathrm{d}}(\lambda)$ 都要高于巴木错, 班公错最小, 说明三者之间格 仁错的非色素颗粒物吸收占总颗粒物吸收的比例最大,班公错最小 (图 $4 \mathrm{~b})$.
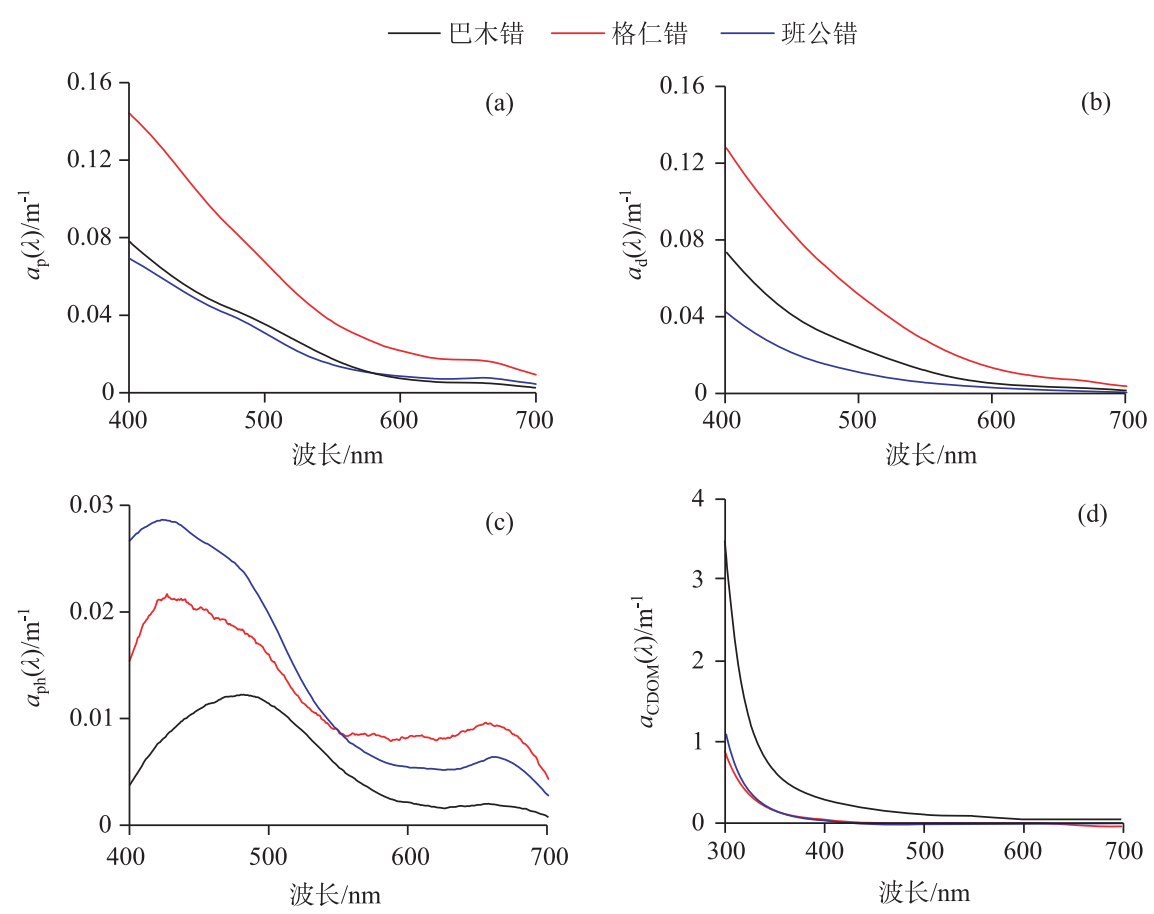

图 4 巴木错、格仁错和班公错光学活性物质的吸收系数

Fig.4 Absorption coefficient of optically constituents in water of Bam Co, Gering Co and Pangong Co

3 个湖泊样本在 440 和 $675 \mathrm{~nm}$ 处 $a_{\mathrm{ph}}(\lambda)$ 均出现吸收峰: 在 $440 \mathrm{~nm}$ 处, $a_{\mathrm{ph}}(\lambda)$ 由高到低分别为班公错、 格仁错和巴木错; 在 $675 \mathrm{~nm}$ 处, $a_{\mathrm{ph}}(\lambda)$ 由高到低分别为格仁错、班公错和巴木错 (图 4c). 班公错较格仁错在 $440 \mathrm{~nm}$ 处的 $a_{\mathrm{ph}}(\lambda)$ 高, 而在 $675 \mathrm{~nm}$ 处低, 而通常认为 $440 \mathrm{~nm}$ 的吸收峰主要由叶绿素 $(\mathrm{a} 、 \mathrm{~b} 、 \mathrm{c}$ ) 和类胡夢卜素 的吸收引起, 而 $675 \mathrm{~nm}$ 的吸收峰主要由 Chl.a 引起 ${ }^{[30]}$, 再对照两者在两处特征波长的峰值变化程度, 特别是 $440 \mathrm{~nm}$ 处, 不难发现班公错的变化要大于格仁错, 说明班公错的藻类颗粒物吸收占自身总吸收的比例较大, 
而 Chl.a 的吸收比格仁错少, 这与 $a_{\mathrm{p}}(\lambda)$ 的分析结果一致. 巴木错的 $a_{\mathrm{ph}}(\lambda)$ 整体较低, 说明其反射率较高, 同 时, 在 $675 \mathrm{~nm}$ 处吸收峰不明显, 且 $440 \mathrm{~nm}$ 的吸收峰向长波方向移动, 说明藻类颗粒物在巴木错中所占比例 较小,与其他两者相比,巴木错水体所含大分子颗粒物较多.

2.2.4 光学活性物质吸收对总吸收的贡献 上文分析中发现不同光学活性物质的吸收特性在不同的采样湖 泊表现出明显的空间差异. 通过观察各个湖库的 CDOM 吸收系数 (图 4d), 发现在常用的 $440 \mathrm{~nm}$ 处许多湖 库的吸收接近于 0 , 吸收非常弱, 为了更好地与其他光学活性物质的吸收特性进行对比分析, 我们选取 400 $\mathrm{nm}$ 作为参考波长, 对有完整测定数据的巴木错、格仁错和班公错样本进行分析, 比较三者不同组分的吸收 在总吸收中所贡献的比例 (图 5), 寻求其影响总吸收的主导因素. 比较分析发现,班公错属于非色素颗粒物 吸收主导的类型, 藻类颗粒物与 CDOM 吸收贡献次之, 藻类颗粒物比 CDOM 贡献略高; 格仁错属于非色素颗 粒物吸收占绝对主导的类型, CDOM 次之, 藻类吸收较少; 巴木错属于 CDOM 吸收占绝对主动的类型, 非色 素颗粒物次之, 藻类颗粒物贡献最少, 在 $400 \mathrm{~nm}$ 处的吸收接近于 0 . 这与之前对各光学活性物质吸收系数的 单独分析结果相符.

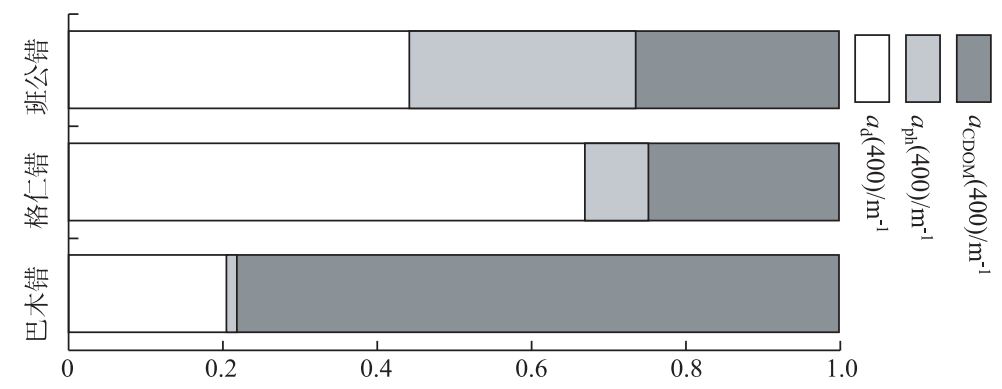

图 5 巴木错、格仁错和班公错水体中各光学活性物质对总吸收的贡献比例

Fig. 5 Contribution of various optically active constituents to the total absorption in water of Bam Co, Gering Co and Pangong Co

\section{$2.3 K_{\mathrm{d}}(\mathrm{PAR})$ 与透明度的相关性分析}

透明度是能够有效表示光在水中衰减的间接指标, 一般透明度较高意味着光在水体中衰减较慢、衰减 系数较小, 而透明度较低, 则意味着光在水体中衰减较快、衰减系数较大. 该地区透明度为 $5.540 \pm 2.660 \mathrm{~m}$. 所以透明度与 $K_{\mathrm{d}}(\mathrm{PAR})$ 都受到水体中各种光学活性物质的影响, 都能够表示光在水体中的衰减程度. 透明 度与 $K_{\mathrm{d}}(\mathrm{PAR})$ 的相关性经前人研究可以表示为 ${ }^{[32]}$ :

$$
K_{\mathrm{d}}(\mathrm{PAR})=f / \mathrm{SD}
$$

不同学者在研究时发现, 针对不同的研究区, $f$ 可以有不同的取值, 根据公式 (7) 建立所有采样点透明 度与 $K_{\mathrm{d}}(\mathrm{PAR})$ 的关系 (图 6a), 通过线性拟合结果发现透明度与 $K_{\mathrm{d}}(\mathrm{PAR})$ 的相关性良好, $R^{2}$ 达到了 0.9 以 上, 拟合度良好, 拟合得到 $f$ 值为 1.19 .

\section{$2.4 K_{\mathrm{d}}(\mathrm{PAR})$ 与光学活性物质的相关性分析}

2.4.1 $K_{\mathrm{d}}(\mathrm{PAR})$ 与 Chl.a 浓度的相关性分析 建立所有采样点的 $K_{\mathrm{d}}(\mathrm{PAR})$ 与对应 Chl.a 浓度的关系. 需要说 明的是, 虽然对单个湖库采样点数据进行平均求值一定程度上可以降低人为选点主观性因素和异常点的影 响, 但是由于采样湖库数量不多, 为了避免由于样本数量不足造成的拟合收玫效果差, 能够更加接近数据的 真实分布情况, 在本节分析中并未对每个湖泊的 $K_{\mathrm{d}}(\mathrm{PAR})$ 与其他光学活性物质参量进行平均求值.

$K_{\mathrm{d}}(\mathrm{PAR})$ 与其 Chl.a 浓度进行线性拟合后发现 $R^{2}$ 达到 0.5 以上 (图 6b), 采样点湖库的 $K_{\mathrm{d}}(\mathrm{PAR})$ 与 Chl.a 之间具有一定的相关性.

2.4.2 $K_{\mathrm{d}}(\mathrm{PAR})$ 与总悬浮颗粒物的相关性分析 $K_{\mathrm{d}}(\mathrm{PAR})$ 与其总悬浮颗粒物浓度 $(\mathrm{TSM})$ 进行线性拟合后发 现, 采样点的 TSM 与其 $K_{\mathrm{d}}(\mathrm{PAR})$ 并没有明显的相关性, $R^{2}$ 只有 0.23 (图 6c).

2.4.3 $K_{\mathrm{d}}(\mathrm{PAR})$ 与 $\mathrm{CDOM}$ 的相关性分析 对 $K_{\mathrm{d}}(\mathrm{PAR})$ 与采样点 CDOM 在 $355 \mathrm{~nm}$ 处的吸收系数 $a_{\mathrm{CDOM}}(355)$ 
进行线性拟合, 发现 $K_{\mathrm{d}}(\mathrm{PAR})$ 与 CDOM 之间的相关性较强, $R^{2}$ 达到了 0.78(图 6d). 综合 $K_{\mathrm{d}}(\mathrm{PAR})$ 与 TSM、 Chl. a 的相关性分析, 可以看出相较于 TSM 和 Chl. a, $K_{\mathrm{d}}(\mathrm{PAR})$ 与 CDOM 的相关性最强, 与有学者对波罗的海 的研究结果 ${ }^{[33]}$ 一致, 但这也并不表示影响所有湖库 $K_{\mathrm{d}}(\mathrm{PAR})$ 值高低的主要因素是 CDOM. 由于自然构造和 人为因素等诸多影响, 导致湖库之间水体光学特性有所差异, 此次采样点分布于青藏高原多个地区, 空间差 异性更加明显, 所以每个湖库的光学特性都会受到某一种或者多种光学活性物质的影响, 不同湖库中占据 主要贡献地位的物质都会有所差异.
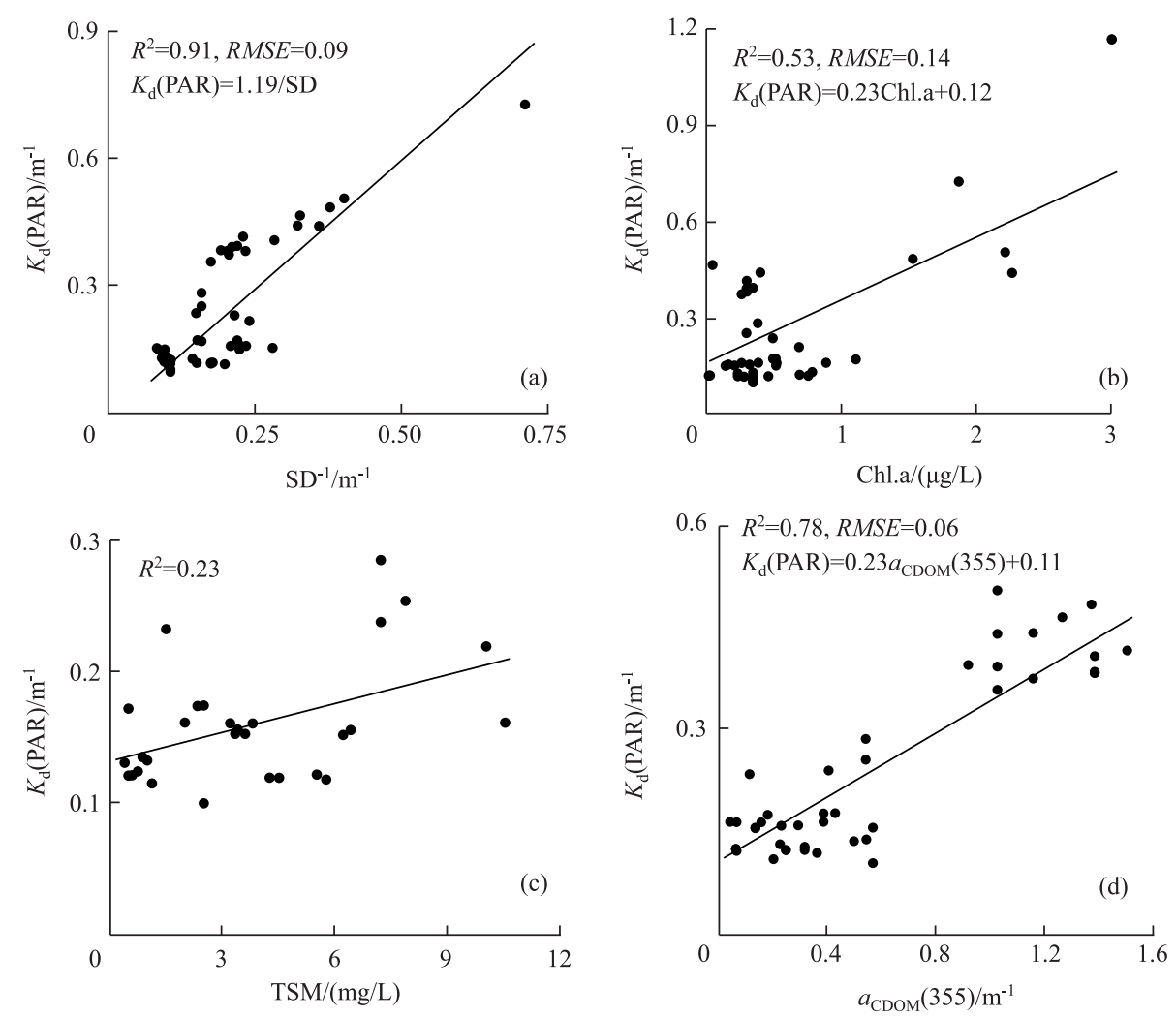

图 $6 K_{\mathrm{d}}(\mathrm{PAR})$ 与透明度 (a)、Chl.a (b)、TSM (c) 和 $a_{\text {CDOM }}(355)$ (d) 的线性拟合

Fig.6 Correlations between $K_{\mathrm{d}}(\mathrm{PAR})$ and SD (a), Chl.a (b), TSM (c) and $a_{\text {CDoM }}(355)$ (d)

\section{3 结论}

由于本研究受到恶劣的自然环境条件和样本运输损失的影响, 导致并未能完整测得所有样点的光学活 性物质吸收特性及含量, 但通过现有数据仍然得到以下结论:

1) 青藏高原地区受到人类活动影响相对较小, 湖库水体整体呈现高透明度、低 $K_{\mathrm{d}}(\mathrm{PAR})$ 的特征. 采样 湖泊整体 $K_{\mathrm{d}}(\mathrm{PAR})$ 为 $0.260 \pm 0.197 \mathrm{~m}^{-1}$; 透明度为 $5.540 \pm 2.660 \mathrm{~m}$.

2) 采样湖库的光学活性物质吸收特性方面: 总体没有呈现出显著的藻类吸收特性, 在 $440 、 550$ 和 $675 \mathrm{~nm}$ 处吸收峰、谷不明显; 非色素颗粒物与 CDOM 吸收特征较藻类颗粒物显著. 巴木错、格仁错和班公错 3 个采 样湖泊中, 占据总颗粒物吸收主导地位的因素各不相同: 班公错属于非色素颗粒物吸收主导的类型, 藻类颗 粒物与 CDOM 吸收贡献次之, 藻类颗粒物比 CDOM 贡献略高; 格仁错属于非色素颗粒物吸收占绝对主导的 类型, CDOM 次之, 藻类吸收较少; 巴木错属于 CDOM 吸收占绝对主动的类型, 非色素颗粒物次之, 藻类颗粒 物贡献最少. 
$3)$ 采样湖库的 $K_{\mathrm{d}}(\mathrm{PAR})$ 实测值与透明度相关性高, 拟合得到的常数 $f$ 较其他水体小, 为 1.19 .

$4) K_{\mathrm{d}}(\mathrm{PAR})$ 与 $\mathrm{CDOM}$ 的相关性较强, 即青藏高原采样湖库 CDOM 含量和分布情况对 $K_{\mathrm{d}}(\mathrm{PAR})$ 解释得 更好, 与 Chl.a 浓度的相关性次之, 与 TSM 没有明显的相关性.

\section{4 参考文献}

[ 1 ] Kirk JTO ed. Light and photosynthesis in aquatic ecosystems: 2nd. Cambridge: Cambridge University Press, $1994: 21$.

[ 2 ] Chen SP, Tong QX, Guo HD eds. Remote sensing information mechanism research. Beijing: Science Press, 1998: 123 [陈述彭, 童庆禧, 郭华东. 遥感信息机理研究. 北京: 科学出版社, 1998: 123].

[ 3 ] Zhang YL, Qin BQ, Chen WM et al. Analysis on distribution and variation of beam attenuation coefficient of Taihu Lake's water. Advances in Water Science, 2003, (4) : 347-353. [张运林, 秦伯强, 陈伟民等. 太湖水体光学衰减系数的分布 及其变化特征. 水科学进展, 2003, (4) : 347-353.]

[ 4 ] Zhang YL. Progress and prospect in lake optics: A review. J Lake Sci, 2011, 23(4) : 483-497. DOI: 10.18307/2011. 0401. [张运林. 湖泊光学研究进展及其展望. 湖泊科学, 2011, 23(4) : 483-497.]

[ 5 ] Zhang YL, Feng S, Ma RH et al. Spatial pattern of euphotic depth and estimation of phytoplankton primary production in Lake Taihu in autumn 2004. J Lake Sci, 2008, 20(3) : 380-388. DOI: 10.18307/2008.0319. [张运林, 冯胜, 马荣华 等. 太湖秋季真光层深度空间分布及浮游植物初级生产力的估算. 湖泊科学, 2008, 20(3) : 380-388.]

[6] Xie GD, Lu CX, Leng YF et al. Ecological assets valuation of the Tibetan plateau. Journal of Natural Resources, 2003, (2) : 189-196. [谢高地, 鲁春霞, 冷允法等. 青藏高原生态资产的价值评估. 自然资源学报, 2003, (2) : 189-196.]

[ 7 ] Wei ZG, Huang RH, Dong WJ. The interannual and interdecadal variability of temperature and precipitation in QinghaiTibet plateau. Chinese Journal of Atomosphere Sciences, 2003, (2) : 157-170. [韦志刚, 黄荣辉, 董文杰. 青藏高原气 温和降水的年际和年代际变化. 大气科学, 2003, (2) : 157-170.]

[ 8 ] Yao TD, Zhu LP. The response of environmental changes on Tibetan Plateau to global changes and adaptation strategy. Advances in Earth Science, 2006, (5): 459-464. [姚檀栋, 朱立平. 青藏高原环境变化对全球变化的响应及其适应对 策. 地球科学进展, 2006, (5): 459-464.]

[ 9 ] Wang SM, Dou HS eds. Chinese lakes. Beijing: Science Press, 1998: 244. [王苏民, 窦鸿身. 中国湖泊志. 北京: 科 学出版社, 1998: 244.]

[10] Jiang YJ, Li SJ, Shen DF et al. Climate change and its impact on the lake environment in the Tibetan Plateau in 19712008. Scientia Geographica Sinica, 2012, (12) : 1503-1512. [姜永见, 李世杰, 沈德福等. 青藏高原近 40 年来气候 变化特征及湖泊环境响应. 地理科学, 2012, (12): 1503-1512.]

[11] Qiao C, Luo JC, Sheng YW et al. Analysis on lake changes since ancient and modern ages using remote sensing in dagze Co, Tibetan Plateau. J Lake Sci, 2010, 22(1): 98-102. DOI: 10.18307/2010.0114. [乔程, 骆剑承, 盛永伟等. 青藏 高原湖泊古今变化的遥感分析一以达则错为例. 湖泊科学, 2010, 22(1): 98-102.]

[12] Zhang SP, Zhang HC, Chen GJ et al. Climate and Glacier changes and lake response in the Ngangla ringsto catchment in Western Tibetan Plateau. Journal of Glaciology and Geocryology, 2012, 22(2) : 267-276. [张淑萍, 张虎才, 陈光杰等. 1973-2010 年青藏高原西部昂拉仁错流域气候、冰川变化与湖泊响应. 冰川冻土, 2012, 22(2) : 267-276.]

[13] Zhang YL, Qin BQ, Hu WP et al. Temporal-spatial variations of euphotic depth of typical lake regions in Lake Taihu and its ecological environmental significance. Science in China Series D-Earth Sciences, 2006, 49(4) : 431-442. DOI: 10. 1007/s11430-006-0431-4.

[14] Devlin MJ, Barry J, Mills DK et al. Estimating the diffuse attenuation coefficient from optically active constituents in UK marine waters. Estuarine Coastal and Shelf Science, 2009, 82(1) : 73-83. DOI: 10.1016/j.ecss.2008.12.015.

[15] Lee ZP, Shang SL, Hu CM et al. Secchi disk depth: A new theory and mechanistic model for underwater visibility. Remote Sensing of Environment, 2015, 169: 139-149. DOI: 10.1016/j.rse.2015.08.002.

[16] Sun DY, Qiu ZF, Li YM et al. New strategy to improve estimation of diffuse attenuation coefficient for highly turbid inland waters. International Journal of Remote Sensing, 2014, 35(9) : 3350-3371. DOI: 10.1080/01431161.2014.904972.

[17] Zhang YL, Liu XH, Yin Y et al. A simple optical model to estimate diffuse attenuation coefficient of photosynthetically active radiation in an extremely turbid lake from surface reflectance. Optics Express, 2012, 20(18) : 20482-20493. DOI: 10. 
1364/OE.20.020482.

[18] Li J, Li MH, Fang XM et al. Hydrochemical characteristics of the Hurleg Lake. Arid Land Geography, 2015, (1) : 43-51. [李晈, 李明慧, 方小敏等. 柯鲁克湖水化学特征分析. 干旱区地理, 2015, (1) : 43-51.

[19] Liu XX, Wen ZH, Shu LC et al. Analysis of surface area changes of Keluke and tuosu lakes over past 40 years and influencing factors. Water Resources Protection, 2014, (1) : 28-33, 63. [刘晓雪, 温忠辉, 束龙仓等. 近 40 年可鲁克湖-托 素湖面积变化及影响因素分析. 水资源保护, 2014, (1):28-33, 63.]

[20] Ju JT, Zhu LP, Wang Y et al. Composition, spatial distribution and environmental significance of water ions in Lake Pumayum Co and its catchment, Southern Tibet. J Lake Sci, 2008, 20(5) : 591-599. DOI: 10.18307/2008.0507. [鞠建廷, 朱立平, 汪勇等. 藏南普莫雍错流域水体离子组成与空间分布及其环境意义. 湖泊科学, 2008, 20(5) : 591-599.]

[21] Ju JT, Zhu LP, Feng JL et al. Granularity reveal the Qinghai-Tibet plateau lakes hydrodynamic modern process: in southern Tibet MoYong lake. Chinese Science Bulletin, 2012, (19): 1781-1790. [鞠建廷, 朱立平, 冯金良等. 粒度揭示的 青藏高原湖泊水动力现代过程: 以藏南普莫雍错为例. 科学通报, 2012, (19)：1781-1790.]

[22] Ma JH. Spatial pattern of Kd(PAR) for waters in Northeast China and its main regulating factors[Dissertation]. Beijing: University of Chinese Academy of Sciences, 2016. [马建行. 东北地区典型湖库 Kd(PAR) 与真光层深度遥感反演 [学 位论文]. 北京: 中国科学院大学, 2016.]

[23] Li WH. Retrieval of euphotic zone depth of Jin River by remote sensing technology. Journal of Subtrophical Resources and Environment, 2009, (3) : 64-72. [李婉晖. 晋江水体真光层深度遥感反演. 亚热带资源与环境学报, 2009, (3) : 64-72. ]

[24] Gi RNM, Zhuo G. The preliminary study of radiation attenuation characteristics in Namtso lake. Journal of Tibet University, 2012, (1): 11-14. [次仁尼玛, 卓嘎. 纳木措水体中辐射衰减特性的初步研究. 西藏大学学报: 自然科学版, 2012, (1): 11-14.]

[25] Bai XH, Cao T, Ni LY. Seasonal variation of water column optical parameters in Lake Erhai, China. Journal of Hydroecol$o g y, 2016,(2): 10-16$. [摆晓虎, 曹特, 倪乐意等. 洱海水体光学特性的季节变化及其影响因素分析. 水生态学 杂志, 2016, (2) : 10-16.]

[26] Li SJ. Absorption characteristics of optically active constituents (OACs) and their remote retrieval based on Quasi-Analytical Alogorithm ( QAA) [Dissertation]. Siping: Jilin Normal University, 2015. [李思佳. 内陆水体光学活性物质吸收特 性与 QAA 反演[学位论文]. 四平: 吉林师范大学, 2015.]

[27] Shao TT, Song KS, Ding Z et al. Absorption characteristics and seasonal variations of optically active water constituents from Liaohe River. Acta Ecologica Sinica, 2016, (7): 1861-1871. [邵田田, 宋开山, 丁智等. 辽河水体光学吸收特 性的季节变化. 生态学报, 2016, (7) : 1861-1871.]

[28] Wang X. Remote sensing of water optical properties and the suspended material concentration in northern Taihu lake [Dissertation ]. Nanjing: Nanjing University of Information Science and Technology, 2007. [王全金. 太湖北部湖区水体光学特 性及悬浮物浓度遥感反演 [学位论文]. 南京: 南京信息工程大学, 2007.]

[29] Dai YN, Li SJ, Wang XJ. Measurement and analysis on the apparent optical properties of water in Chaohu Lake. Environmental Science of China, 2008, (11): 979-983. [戴永宁, 李素菊, 王学军. 巢湖水体的表观光学特性测量与分析. 中国环境科学, 2008, (11): 979-983.]

[30] Li SJ, Song KS, Chen ZW et al. Absorption characteristics of particulates and CDOM in spring in the Lake Xingkai. J Lake Sci, 2015, 27 (5) : 941-952. DOI: 10.18307/2015.0522. [李思佳, 宋开山, 陈智文等. 兴凯湖春季水体悬浮颗粒物 和 CDOM 吸收特性. 湖泊科学, 2015, 27(5): 941-952.]

[31] Shao TT, Song KS, Du J et al. Seasonal variations of CDOM optical properties in rivers across the Liaohe Delta. Wetlands, 2016, 36: S181-S192. DOI: 10.1007/s13157-014-0622-2.

[32] Robert WH. The secchi disk in turbid coastal waters1. Limnology and Oceanography, 1970, 15(5) : 688-694.

[33] Kratzer S, Hakansson B, Sahlin C. Assessing Secchi and photic zone depth in the Baltic Sea from satellite data. Ambio, 2003, 32(8) : 577-585. DOI: 10.1579/0044-7447-32.8.577. 